\title{
An Analytical Method for Jack-Up Riser's Fatigue Life Estimation
}

\author{
Fengde Wang $\mathbb{D}^{1},{ }^{1}$ Wensheng Xiao, ${ }^{1}$ Qi Liu, ${ }^{1}$ Lei Wu ${ }^{D},{ }^{1}$ and Zhanbin Meng ${ }^{2}$ \\ ${ }^{1}$ College of Mechanical and Electronic Engineering, China University of Petroleum (East China), Qingdao, Shandong 266580, China \\ ${ }^{2}$ Drilling Technology Research Institute of Shengli Oilfield, Dongying, Shandong 257061, China \\ Correspondence should be addressed to Fengde Wang; b14040125@s.upc.edu.cn
}

Received 30 November 2017; Accepted 29 March 2018; Published 10 May 2018

Academic Editor: Francesco Pellicano

Copyright (c) 2018 Fengde Wang et al. This is an open access article distributed under the Creative Commons Attribution License, which permits unrestricted use, distribution, and reproduction in any medium, provided the original work is properly cited.

\begin{abstract}
In order to determine whether a special sea area and its sea state are available for the jack-up riser with surface blowout preventers, an analytical method is presented to estimate the jack-up riser's wave loading fatigue life in this study. In addition, an approximate formula is derived to compute the random wave force spectrum of the small-scale structures. The results show that the response of jack-up riser is a narrow band random vibration. The infinite water depth dispersion relation between wavenumber and wave frequency can be used to calculate the wave force spectrum of small-scale structures. The riser's response mainly consists of the additional displacement response. The fatigue life obtained by the formula proposed by Steinberg is less than that of the Bendat method.
\end{abstract}

\section{Introduction}

The jack-up platform often uses surface blowout preventers (BOP) to drill exploratory well in shallow sea (under $90 \mathrm{~m}$ water depth, as shown in Figure 1). This kind of drilling process is widely used due to its low cost. However, as the mainstream jack-up platform can be operated in $122 \mathrm{~m}$ water depth, the lateral rigidity of the platform decreases with the increase of water depth, which makes the platform in a random vibration status. The platform's vibration can reduce the drilling riser's service life. Therefore, it is necessary to study the random response of the jack-up riser.

In this study, we choose the jack-up riser as the research object. The riser is modelled as a Bernoulli-Euler beam, the joint between the riser and the platform can be regarded as a hinge, and the riser's lower end is clamped on the seabed. The platform's vibration could be treated as the riser's timedependent boundary condition, and the riser bears the axial compressive load resulting from surface BOP. Thus, the jackup riser is modelled as an axial loaded Bernoulli-Euler beam with time-dependent boundary condition.

Many researches have been done on the fatigue analysis of marine riser. Trim et al. [1] introduced a series of experiments on riser models over a range of scales and current conditions; their research results suggest that a key consideration in vortex-induced vibration (VIV) fatigue design is the length of suppression coverage, as well as the nature of the flow to which the bare section of the riser is exposed. Based on the energy equilibrium theory and the forced vibration experimental data of a rigid cylinder, Xue et al. [2] presented a prediction model of VIV fatigue damage for riser accounting for both cross-flow and in-line vibrations. Considering parametric excitations, Zhang and Tang [3] investigated VIV fatigue analysis of deep-water risers in shear flow. Low and Srinil [4] made a VIV fatigue reliability analysis of marine risers with uncertainties in the wake oscillator model. $\mathrm{Xu}$ et al. [5] proposed a quick and accurate VIV model to predict the fatigue life of marine drilling risers. Low [6] extended a time/frequency domain hybrid method for the fatigue analysis of risers, and the linearized frequency domain approach is proven to be adequate in mild sea states. Li and Low [7] studied the influence of low-frequency vessel motions on the fatigue response of steel catenary risers at the touchdown point. Elosta et al. [8] studied the fatigue performance's sensitivity to geotechnical parameters with a parametric study. Low [9] proposed a variance reduction approach for the long-term fatigue analysis of offshore structures by Monte Carlo simulation. As for beam's 


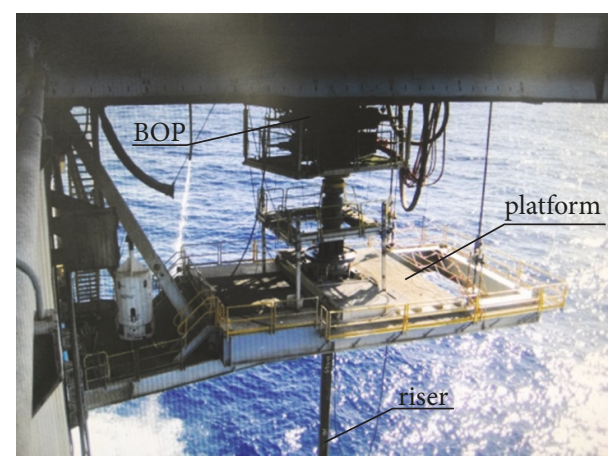

FIGURE 1: Real work conditions of the jack-up riser with surface BOP.

dynamic analysis, the vibratory response of a beam with timedependent boundary conditions can be obtained by Laplace transform [10,11] and the Mindlin-Goodman method [1216]. In the Mindlin-Goodman method, the nonhomogeneous boundary conditions are transformed into homogeneous ones. Therefore, the method of separation of variables can be used to solve the beam's response [17]. Besides, the influence of axial load on the dynamics of structures has attracted much attention as a result of its wide applications [18-20]. However, only few researches on the jack-up riser's fatigue life estimation can be found in literature; and it is necessary to determine the riser's random response before drilling an oil well in the deep-shallow sea. The riser's fatigue life can be applied as a quantitative criterion to judge whether a jackup riser with surface BOP can be used in a special sea area. Therefore, we propose an analytical method to estimate the jack-up riser's wave loading fatigue life by the MindlinGoodman method and the Steinberg method in frequency domain.

The current paper is organized as follows. Firstly, the mathematical model is established in Section 2. And then the analytical procedure to solve the problem is presented in Section 3. Subsequently, a case study is carried out in Section 4. Finally, several conclusions are summarized in Section 5 .

\section{Mathematical Model}

Since the jack-up riser has characteristics of small inclination angle and little deformation, its lateral motion is governed by the following equation $[3,5]$ :

$$
\begin{aligned}
& E I \frac{\partial^{4} y(x, t)}{\partial x^{4}}+N(x) \frac{\partial^{2} y(x, t)}{\partial x^{2}}+m \frac{\partial^{2} y(x, t)}{\partial t^{2}} \\
& +c \frac{\partial y(x, t)}{\partial t}=0 .
\end{aligned}
$$

In (1), $t$ is the time, $x$ is the coordinate measured along the axis of riser, $y(x, t)$ is the transverse deflection of the beam axis, $E$ is the modulus of elasticity, $I$ is the area moment of inertia, $N(x)$ is the beam with an axial compressive force, $m$ is the mass per unit length, and $c$ is the damping coefficient. In this study, to simplify the computational process, the axial force at the riser's midpoint is used as the average axial force $N$ [5].

Equation (2) presents the riser's time-dependent boundary conditions at $x=0$ and $x=l$ :

$$
\begin{array}{ll}
\frac{\partial y(0, t)}{\partial x}=0 & y(0, t)=0 ; \\
\frac{\partial y^{2}(l, t)}{\partial x^{2}}=0 & y(l, t)=u(t),
\end{array}
$$

where $l$ is the length between the riser's clamped end and hinged end and $u(t)$ is the random vibration response of the platform. The wave is a stationary Gaussian random process and the jack-up platform can be modelled as a linear system. By the Morison equation and the linearization method proposed by Borgman [21], $u(t)$ can be treated as a stationary Gaussian random process.

The beam's transverse deflection $y(x, t)$ is decomposed into two parts according to the Mindlin-Goodman method: one is quasi-static displacement $y_{s}(x, t)$ resulting from the platform vibration and the other one is additional displacement $y_{d}(x, t)$ due to the dynamic inertial force [22].

$$
\begin{aligned}
& y(x, t)=y_{s}(x, t)+y_{d}(x, t), \\
& y_{s}(x, t)=\sum_{i=1}^{r} g_{i}(x) u_{i}(t), \\
& y_{d}(x, t)=\sum_{n=1}^{\infty} \varphi_{n}(x) q_{n}(t),
\end{aligned}
$$

where $g_{i}(x)$ is static influence function, $\varphi_{n}(x)$ is the shape function of the riser, and $q_{n}(t)$ is the modal coordinate of $i$ th mode. The boundary conditions of $y_{s}(x, t)$ and $y_{d}(x, t)$ are formulated by the following equations:

$$
\begin{array}{ll}
\frac{\partial y_{s}(0, t)}{\partial x}=0 & y_{s}(0, t)=0 \\
\frac{\partial y_{s}^{2}(l, t)}{\partial x^{2}}=0 & y_{s}(l, t)=u(t) \\
\frac{\partial y_{d}(0, t)}{\partial x}=0 & y_{d}(0, t)=0 \\
\frac{\partial y_{d}^{2}(l, t)}{\partial x^{2}}=0 & y_{d}(l, t)=0
\end{array}
$$

The index $r$ is determined by the boundary conditions; for the jack-up riser, $r=1$, and $g_{i}(x)$ can be obtained by initial parametric method [22].

$$
g(x)=\frac{3 x^{2}}{2 l^{2}}-\frac{x^{3}}{2 l^{3}}
$$

Substituting (3) into (1),

$$
\begin{aligned}
& E I \frac{\partial^{4} y_{d}}{\partial x^{4}}+N \frac{\partial^{2} y_{d}}{\partial x^{2}}+m \frac{\partial^{2} y_{d}}{\partial t^{2}}+c \frac{\partial y_{d}}{\partial t}=F_{\mathrm{eq}} \\
& F_{\mathrm{eq}}=-E I \frac{\partial^{4} y_{s}}{\partial x^{4}}-N \frac{\partial^{2} y_{s}}{\partial x^{2}}-m \frac{\partial^{2} y_{s}}{\partial t^{2}}-c \frac{\partial y_{s}}{\partial t},
\end{aligned}
$$

where $F_{\text {eq }}$ is the equivalent load. 
As a result of (8), the first item's value in the right side of (10) is zero; therefore, (10) can be rewritten as

$$
F_{\mathrm{eq}}=-N \frac{\partial^{2} y_{s}}{\partial x^{2}}-m \frac{\partial^{2} y_{s}}{\partial t^{2}}-c \frac{\partial y_{s}}{\partial t}
$$

The frequency equation of the riser's lateral vibration is derived as

$$
\begin{aligned}
\sqrt{\beta^{2}+\eta} \cdot \tanh (\beta l) & =\beta \tan \left(l \sqrt{\beta^{2}+\eta}\right), \\
\beta & =\sqrt{\left(\lambda^{4}+\frac{\eta^{2}}{4}\right)^{1 / 2}-\frac{\eta}{2} .}
\end{aligned}
$$

In (13), $\beta$ represents solutions of (12), $\lambda$ is frequency coefficient, and $\eta$ is axial force impact factor.

$$
\begin{gathered}
\lambda^{4}=\frac{m \omega^{2}}{E I} \\
\eta=\frac{N}{E I}
\end{gathered}
$$

where $\omega$ is circular frequency.

Mode functions of the riser's lateral vibration are formulated by the following equation:

$$
\begin{aligned}
\varphi_{n}(x)= & \sin \lambda_{n} x-\sinh \lambda_{n} x+\frac{\sin \lambda_{n} l+\sinh \lambda_{n} l}{\cos \lambda_{n} l+\cosh \lambda_{n} l} \\
& \times\left(\cosh \lambda_{n} x-\cos \lambda_{n} x\right), \\
& \quad(n=1,2, \ldots, \infty) .
\end{aligned}
$$

\section{Analysis}

3.1. Random Vibration Analysis of the Jack-Up Platform. As the lateral vibration of the riser is mainly induced by the platform's horizontal vibration, the response of the platform has to be calculated firstly.

According to the rules of the China Classification Society (CCS), we choose the single degree of freedom model to describe jack-up platform. Therefore, the motion of the platform can be formulated by the following equation:

$$
m_{e} \ddot{u}(t)+c \dot{u}(t)+k_{e} u(t)=p(t),
$$

where $m_{e}$ is equivalent mass of the platform, $k_{e}$ is equivalent bending stiffness, and $p(t)$ is random wave load.

The frequency response function of the platform is formulated as

$$
T(\omega)=\frac{1}{-m_{e} \omega^{2}+j c \omega+k_{e}} .
$$

In this study, Pierson-Moskowitz spectrum is used to model the wave.

$$
S(\omega)=\frac{0.78}{\omega^{5}} \exp \left(-\frac{3.11}{\omega^{4} H_{s}^{2}}\right) .
$$

In (18), $S(\omega)$ is Pierson-Moskowitz spectrum, and $H_{s}$ is the significant wave height.

According to the Morison equation and Borgman's linearization method, the wave force spectrum of one leg at arbitrary height can be obtained by the following equation:

$$
\begin{aligned}
S_{f}(\omega)= & {\left[\frac{1}{2} C_{D} \rho D_{o} \sigma \sqrt{\frac{8}{\pi}} \omega \frac{\cosh (k h)}{\sinh (k d)}\right]^{2} S(\omega) } \\
& +\left[C_{M} \rho \frac{\pi D^{2}}{4} \omega^{2} \frac{\cosh (k h)}{\sinh (k d)}\right]^{2} S(\omega),
\end{aligned}
$$

where $C_{D}$ is drag force coefficient, $C_{M}$ is inertia force coefficient, $k$ is wavenumber, $\rho$ is the density of seawater, $g$ is the gravitational acceleration, $h$ is the coordinate along the leg, $D_{o}$ is leg's equivalent outer diameter, and $d$ is water's depth.

Based on (19), the wave force spectrum of one leg $S_{F}(\omega)$ can be obtained by the integral along the direction of the water depth.

$$
\begin{aligned}
S_{F}(\omega)= & {\left[\frac{C_{D} \rho D_{o} \omega}{\sinh (k d)} \sqrt{\frac{2}{\pi}} \int_{0}^{d} \sigma \cosh (k h) d h\right]^{2} S(\omega) } \\
& +\left[\frac{C_{M} \rho g \pi D^{2}}{4} \tanh (k d)\right]^{2} S(\omega) .
\end{aligned}
$$

In $(20)$,

$$
\sigma^{2}=\int_{0}^{\infty}\left(\omega \frac{\cosh k h}{\sinh k d}\right)^{2} S(\omega) d \omega
$$

Under a certain water depth, $\sigma^{2}$ is the variance of water particle's horizontal speed. Considering the fact that the linearization of the drag force is an approximate treatment, the integral in (21) is calculated by Pierson-Moskowitz spectrum only, and the variance can be obtained as

$$
\sigma \approx 0.25 H_{s} \omega \frac{\cosh (k h)}{\sinh (k d)}
$$

Substituting (22) into (20), the approximate expression of one leg's total wave force spectrum can be obtained as follows:

$$
\begin{aligned}
S_{F}(\omega) & \\
= & \left\{\frac{\left(C_{D} \rho D_{o} g H_{s}\right)^{2}}{32 \pi}\left[\frac{\sinh (2 k d)+2 k d}{\sinh (2 k d)}\right]^{2}\right\} S(\omega) \\
& +\left[\frac{1}{4} C_{M} \rho g \pi D_{o}^{2} \tanh (k d)\right]^{2} S(\omega) .
\end{aligned}
$$

According to the definition of power spectral density function and the relationship between autocorrelation function and power spectral density function, the total wave 
force spectrum of the platform $S_{p}(\omega)$ can be derived by the following equation:

$$
\begin{aligned}
S_{p}(\omega) & \\
= & \left\{\frac{9\left(C_{D} \rho D_{o} g H_{s}\right)^{2}}{32 \pi}\left[\frac{\sinh (2 k d)+2 k d}{\sinh (2 k d)}\right]^{2}\right\} S(\omega) \\
& +\left[\frac{3}{4} C_{M} \rho g \pi D_{o}^{2} \tanh (k d)\right]^{2} S(\omega) .
\end{aligned}
$$

Based on (17) and (24), the random response spectrum of the platform can be obtained as

$$
S_{u}(\omega)=|T(\omega)|^{2} S_{p}(\omega) .
$$

3.2. Random Vibration Analysis of the Jack-Up Riser. Substituting (4) and (5) into (9) generates

$$
\begin{aligned}
E I \sum_{n=1}^{\infty} \frac{d^{4} \varphi_{n}}{d x^{4}} q_{n}(t)+N \sum_{n=1}^{\infty} \frac{d^{2} \varphi_{n}}{d x^{2}} q_{n}(t) \\
\quad+m \sum_{n=1}^{\infty} \varphi_{n}(x) \ddot{q}_{n}(t)+c \sum_{n=1}^{\infty} \varphi_{n}(x) \dot{q}_{n}(t) \\
=-m g(x) \ddot{u}(t)-c g(x) \dot{u}(t)-N \frac{d^{2} g(x)}{d x^{2}} u(t) .
\end{aligned}
$$

It can be deduced from (7) that the additional displacement has the homogeneous boundary condition; therefore, its mode shape functions conform to the following relationship:

$$
E I \frac{d^{4} \varphi_{n}(x)}{d x^{4}}+N \frac{d^{2} \varphi_{n}(x)}{d x^{2}}-m \omega_{n}^{2} \varphi_{n}(x)=0 .
$$
(26).

Equation (28) can be obtained by substituting (27) into

$$
\begin{aligned}
& m \sum_{n=1}^{\infty} \varphi_{n}(x) \ddot{q}_{n}(t)+c \sum_{n=1}^{\infty} \varphi_{n}(x) \dot{q}_{n}(t) \\
& \quad+m \omega_{n}^{2} \sum_{n=1}^{\infty} \varphi_{n}(x) q_{n}(t) \\
& \quad=-m g(x) \ddot{u}(t)-c g(x) \dot{u}(t)-N \frac{d^{2} g(x)}{d x^{2}} u(t) .
\end{aligned}
$$

Then, (28) can be decoupled to (29) by the orthogonality conditions.

$$
\begin{aligned}
& m \ddot{q}_{n}(t)+c \dot{q}_{n}(t)+m \omega_{n}^{2} q_{n}(t) \\
& =-m \delta_{n} \ddot{u}(t)-c \delta_{n} \dot{u}(t)-N \varepsilon_{n} u(t), \\
& \delta_{n}=\frac{\int_{0}^{l} g(x) \varphi_{n}(x) d x}{\int_{0}^{l} \varphi_{n}^{2}(x) d x} \\
& \varepsilon_{n}=\frac{\int_{0}^{l} g^{\prime \prime}(x) \varphi_{n}(x) d x}{\int_{0}^{l} \varphi_{n}^{2}(x) d x}
\end{aligned}
$$

where $\delta_{n}$ and $\varepsilon_{n}$ are weight coefficients of equivalent load.
According to the linear theory, the frequency response function of the $n$ th-order modal coordinates of the riser's additional displacement versus $u(t)$ can be obtained in the following equation:

$$
H_{n}(\omega)=\frac{m \delta_{n} \omega^{2}-j c \delta_{n} \omega-N \varepsilon_{n}}{m \omega_{n}^{2}-m \omega^{2}+j c \omega} .
$$

And then the frequency response function of additional displacement versus $u(t)$ can be obtained by (5) and (31).

$$
H(x, \omega)=\sum_{n=1}^{\infty} \varphi_{n}(x) H_{n}(\omega) \quad(n=1,2, \ldots, \infty) .
$$

Let $S_{d}(x, \omega)$ denote the power spectral density function of $y_{d}(x, t)$, and let $S_{d u}(x, \omega)$ denote the cross power spectral density function between $y_{d}(x, t)$ and $u(t)$. Based on (25) and (32), $S_{d}(x, \omega)$ and $S_{d u}(x, \omega)$ can be obtained as follows:

$$
\begin{gathered}
S_{d}(x, \omega)=S_{u}(\omega)|H(x, \omega)|^{2}, \\
S_{d u}(x, \omega)=S_{u}(\omega) H(x, \omega) .
\end{gathered}
$$

The relationship between displacement and stress can be expressed as

$$
Y(x, t)=\frac{E I}{W} \frac{\partial y^{2}(x, t)}{\partial x^{2}}
$$

where $Y(x, t)$ denotes stress and $W$ is the bending modulus of the riser.

On the basis of (32) and (35), we can get the frequency response function of the riser's stress resulting from $y_{d}(x, t)$ :

$$
\begin{aligned}
H_{Y}(x, \omega)=\frac{E I}{W} \sum_{n=1}^{\infty} H_{n}(\omega) \frac{d^{2}}{d x^{2}} \varphi_{n}(x) & \\
& (n=1,2, \ldots, \infty) .
\end{aligned}
$$

Then, the power spectral density function of stress resulting from $y_{d}(x, t)$ can be obtained by (33) and (36):

$$
\begin{aligned}
& S_{d Y}(x, \omega) \\
& =S_{u}(\omega)\left(\frac{E I}{W}\right)^{2} \sum_{n=1}^{\infty}\left|H_{n}(\omega)\right|^{2}\left[\frac{d^{2}}{d x^{2}} \varphi_{n}(x)\right]^{2} \\
& \quad(n=1,2, \ldots, \infty) .
\end{aligned}
$$

And the cross power spectral density function of the stress can be obtained in the following equation:

$$
\begin{array}{r}
S_{d u Y}(x, \omega)=S_{u}(\omega) \frac{E I}{W} \sum_{n=1}^{\infty} H_{n}(\omega)\left[\frac{d^{2}}{d x^{2}} \varphi_{n}(x)\right] \\
(n=1,2, \ldots, \infty) .
\end{array}
$$

As the definition of autocorrelation function and the Wiener-Khintchine principle, the power spectral density 
TABLE 1: Parameters of nontubular joints' $S$ - $N$ curves in sea water (CCS).

\begin{tabular}{lcc}
\hline Grade & $K$ & $p$ \\
\hline B & $3.37 \times 10^{14}$ & 4.0 \\
C & $1.41 \times 10^{13}$ & 3.5 \\
D & $5.07 \times 10^{11}$ & 3.0 \\
E & $3.47 \times 10^{11}$ & 3.0 \\
F & $2.10 \times 10^{11}$ & 3.0 \\
$F_{2}$ & $1.43 \times 10^{11}$ & 3.0 \\
G & $8.33 \times 10^{10}$ & 3.0 \\
W & $5.33 \times 10^{10}$ & 3.0 \\
\hline
\end{tabular}

functions of the stress resulting from $y_{s}(x, t)$ can be generated in the following equation:

$$
\begin{aligned}
S_{u Y}(x, \omega)=S_{u}(\omega)\left(\frac{E I}{W}\right)^{2}\left[\frac{d^{2}}{d x^{2}} g(x)\right]^{2} & \\
& (n=1,2, \ldots, \infty) .
\end{aligned}
$$

According to the spectrum's summation formula, the power spectral density functions of the riser's bending stress can be obtained by combining (37), (38), and Eq. (39).

$$
\begin{aligned}
S_{Y}(x, \omega)= & S_{d Y}(x, \omega)+2 \operatorname{Re}\left[S_{d u Y}(x, \omega)\right] \\
& +S_{u Y}(x, \omega) .
\end{aligned}
$$

In Eq. (40), $\operatorname{Re}\left[S_{d u Y}(x, \omega)\right]$ denotes the real part of $S_{d u Y}(x, \omega)$.

3.3. Fatigue Life Estimation. Based on the above analysis, the fatigue life estimation is carried out subsequently.

Because the jack-up riser is operated in corrosion environment, the $S-N$ curve of nontubular joints in sea water is applied in this study. The curve is formulated in the following equation:

$$
\log (N)=\log (K)-p \log (S)
$$

where $S$ is stress range, $N$ is cycle number, $K$ is constant, and $p$ is slope; the values of $K$ and $m$ can be selected from Table 1 .

As for narrow band random vibration, Bendat [23] proposed a formula to estimate structures' fatigue life based on the power spectral density function; and the formula is written as follows:

$$
T_{B}=\frac{K}{E[0](\sqrt{2} \sigma)^{m} \Gamma(1+m / 2)} .
$$

In (42), $\Gamma(z)$ is Gamma function, which is formulated in the following equation:

$$
\Gamma(z)=\int_{0}^{\infty} t^{z-1} e^{-t} d t
$$

where $z$ is independent variable.

Steinberg [24] also proposed an empirical equation for the fatigue life estimation based on the assumption that the

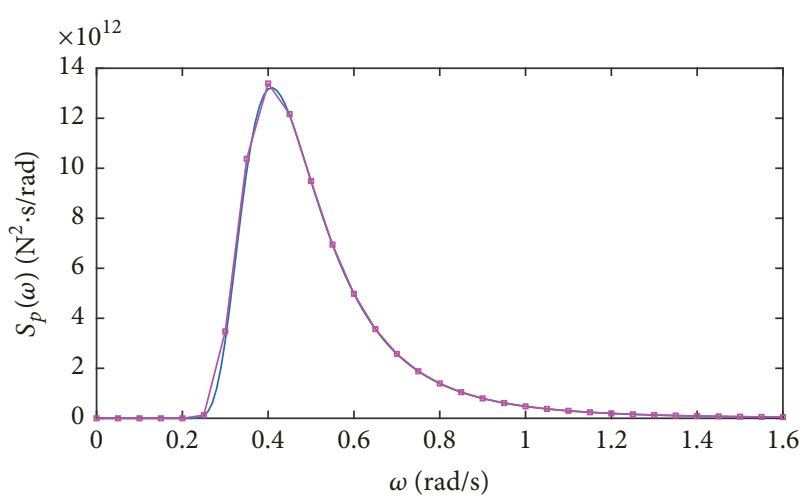

— PSD of wave load under infinite water depth condition

$\simeq$ PSD of wave load under finite water depth condition

FIgURE 2: The power spectral density (PSD) function of wave force under finite water depth condition and infinite water depth condition $\left(H_{s}=10 \mathrm{~m}\right)$.

maximum stress response would not exceed the six times mean square deviation. The equation is presented as

$$
T_{S}=\frac{K}{E[0] \cdot\left[0.683 \zeta^{m}+0.271(2 \zeta)^{m}+0.043(3 \zeta)^{m}\right]} .
$$

\section{Case Study}

Taking a jack-up platform as the example [25], the quantitative research of the riser's fatigue life estimation is carried out by the method proposed in this study. The platform's parameters are listed as follows: $m_{e}=6.48 \times 10^{6} \mathrm{~kg}, k_{e}=$ $4.71 \times 10^{6} \mathrm{~N} / \mathrm{m}, c=8.77 \times 10^{5} \mathrm{~N} \cdot \mathrm{m} / \mathrm{s}, D_{o}=3.62 \mathrm{~m}, E I=$ $8.24 \times 10^{8} \mathrm{~N} \cdot \mathrm{m}^{2}, m=461 \mathrm{~kg} / \mathrm{m}, l=110 \mathrm{~m}, \eta=7 \times 10^{-4} \mathrm{~m}^{-2}$, $H_{s}=10 \mathrm{~m}, C_{D}=2.0, C_{M}=2.0, d=100 \mathrm{~m}, \rho=1025 \mathrm{~kg} / \mathrm{m}^{3}$, and $g=9.8 \mathrm{~m} / \mathrm{s}^{2}$.

When we compute the spectrum of the random wave force, there are two independent variables of wavenumber $k$ and circular frequency $\omega$ in (24). Under the condition of the finite water depth, these two variables have the dispersion relation as

$$
\omega^{2}=g k \tanh (k d)
$$

With the increase of water depth, (45) is simplified into the following equation:

$$
\omega^{2}=g k
$$

Under the finite water depth condition, three transcendental equations need to be solved in the calculation of the platform's power spectral density function. In this study, the power spectral density function of the platform was calculated by (45) and (46), respectively. The data are listed in Table 2 and plotted in Figure 2; the results show that there is a minor error between the finite water depth condition and the infinite water depth condition. Therefore, (46) is accurate enough to calculate platform's wave force spectrum. By calculating the mean square deviation of the random 
TABLE 2: The data of the wave force spectrum calculation $\left(H_{s}=10 \mathrm{~m}\right)$.

\begin{tabular}{|c|c|c|c|c|c|c|}
\hline $\begin{array}{l}\text { Sequence } \\
\text { number }\end{array}$ & $\begin{array}{c}\text { Wave } \\
\text { frequency }\end{array}$ & Wavenumber & {$[\tanh (k d)]^{2}$} & {$\left[\frac{\sinh (2 k d)+2 k d}{\sinh (2 k d)}\right]^{2}$} & $\begin{array}{c}\text { Wave force spectrum } \\
\text { under finite water } \\
\text { depth }\end{array}$ & $\begin{array}{c}\text { Wave force spectrum } \\
\text { under infinite water } \\
\text { depth }\end{array}$ \\
\hline 1 & 0.05 & 0.0016 & 0.0252 & 3.9328 & 0.0000 & 0.0000 \\
\hline 2 & 0.10 & 0.0032 & 0.0958 & 3.7437 & 0.0000 & 0.0000 \\
\hline 3 & 0.15 & 0.0050 & 0.2136 & 3.4259 & 0.0000 & 0.0000 \\
\hline 4 & 0.20 & 0.0069 & 0.3576 & 3.0320 & 0.0000 & 0.0000 \\
\hline 5 & 0.25 & 0.0089 & 0.5061 & 2.6177 & $1.23 \times 10^{11}$ & $1.01 \times 10^{11}$ \\
\hline 6 & 0.30 & 0.0113 & 0.6578 & 2.1811 & $3.48 \times 10^{12}$ & $3.11 \times 10^{12}$ \\
\hline 7 & 0.35 & 0.0141 & 0.7876 & 1.7886 & $1.04 \times 10^{13}$ & $9.87 \times 10^{12}$ \\
\hline 8 & 0.40 & 0.0174 & 0.8840 & 1.4753 & $1.34 \times 10^{13}$ & $1.32 \times 10^{13}$ \\
\hline 9 & 0.45 & 0.0213 & 0.9451 & 1.2552 & $1.22 \times 10^{13}$ & $1.21 \times 10^{13}$ \\
\hline 10 & 0.50 & 0.0258 & 0.9773 & 1.1220 & $9.49 \times 10^{12}$ & $9.46 \times 10^{12}$ \\
\hline 11 & 0.55 & 0.0310 & 0.9919 & 1.0510 & $6.95 \times 10^{12}$ & $6.95 \times 10^{12}$ \\
\hline 12 & 0.60 & 0.0368 & 0.9975 & 1.0188 & $4.98 \times 10^{12}$ & $4.98 \times 10^{12}$ \\
\hline 13 & 0.65 & 0.0431 & 0.9993 & 1.0062 & $3.57 \times 10^{12}$ & $3.57 \times 10^{12}$ \\
\hline 14 & 0.70 & 0.0500 & 0.9998 & 1.0018 & $2.57 \times 10^{12}$ & $2.57 \times 10^{12}$ \\
\hline 15 & 0.75 & 0.0574 & 1.0000 & 1.0005 & $1.88 \times 10^{12}$ & $1.88 \times 10^{12}$ \\
\hline 16 & 0.80 & 0.0653 & 1.0000 & 1.0001 & $1.39 \times 10^{12}$ & $1.39 \times 10^{12}$ \\
\hline 17 & 0.85 & 0.0737 & 1.0000 & 1.0000 & $1.05 \times 10^{12}$ & $1.05 \times 10^{12}$ \\
\hline 18 & 0.90 & 0.0827 & 1.0000 & 1.0000 & $7.96 \times 10^{11}$ & $7.96 \times 10^{11}$ \\
\hline 19 & 0.95 & 0.0921 & 1.0000 & 1.0000 & $6.13 \times 10^{11}$ & $6.13 \times 10^{11}$ \\
\hline 20 & 1.00 & 0.1020 & 1.0000 & 1.0000 & $4.77 \times 10^{11}$ & $4.77 \times 10^{11}$ \\
\hline 21 & 1.05 & 0.1125 & 1.0000 & 1.0000 & $3.76 \times 10^{11}$ & $3.76 \times 10^{11}$ \\
\hline 22 & 1.10 & 0.1235 & 1.0000 & 1.0000 & $2.99 \times 10^{11}$ & $2.99 \times 10^{11}$ \\
\hline 23 & 1.15 & 0.1349 & 1.0000 & 1.0000 & $2.41 \times 10^{11}$ & $2.41 \times 10^{11}$ \\
\hline 24 & 1.20 & 0.1469 & 1.0000 & 1.0000 & $1.95 \times 10^{11}$ & $1.95 \times 10^{11}$ \\
\hline 25 & 1.25 & 0.1594 & 1.0000 & 1.0000 & $1.59 \times 10^{11}$ & $1.59 \times 10^{11}$ \\
\hline 26 & 1.30 & 0.1724 & 1.0000 & 1.0000 & $1.31 \times 10^{11}$ & $1.31 \times 10^{11}$ \\
\hline 27 & 1.35 & 0.1860 & 1.0000 & 1.0000 & $1.09 \times 10^{11}$ & $1.09 \times 10^{11}$ \\
\hline 28 & 1.40 & 0.2000 & 1.0000 & 1.0000 & $9.09 \times 10^{10}$ & $9.08 \times 10^{10}$ \\
\hline 29 & 1.45 & 0.2145 & 1.0000 & 1.0000 & $7.63 \times 10^{10}$ & $7.63 \times 10^{10}$ \\
\hline 30 & 1.50 & 0.2296 & 1.0000 & 1.0000 & $6.45 \times 10^{10}$ & $6.45 \times 10^{10}$ \\
\hline 31 & 1.55 & 0.2452 & 1.0000 & 1.0000 & $5.47 \times 10^{10}$ & $5.48 \times 10^{10}$ \\
\hline 32 & 1.60 & 0.2612 & 1.0000 & 1.0000 & $4.67 \times 10^{10}$ & $4.68 \times 10^{10}$ \\
\hline
\end{tabular}

stress response, an approximate calculation formula for the platform's wave force spectrum can be obtained as follows:

$$
\begin{aligned}
& S_{p}(\omega) \\
& =\left\{\frac{9\left(C_{D} \rho D_{o} g H_{s}\right)^{2}}{32 \pi}\left[\frac{\sinh \left(2 d \omega^{2} / g\right)+2 d \omega^{2} / g}{\sinh \left(2 d \omega^{2} / g\right)}\right]^{2}\right\} \\
& \cdot S(\omega)+\left[\frac{3}{4} C_{M} \rho g \pi D_{o}^{2} \tanh \left(d \omega^{2} / g\right)\right]^{2} S(\omega) .
\end{aligned}
$$

The power spectral density function of the platform's lateral displacement can be obtained by (25) and (47) and is plotted in Figure 3. The results show that the power spectral density function of the platform has two peak frequencies, which are wave force spectrum's dominant frequency and the platform's natural frequency; and the frequency band of the platform is mainly between these two peak frequencies.

In order to get the response of the riser, we have to calculate the riser's natural frequencies and weight coefficients of equivalent load firstly. In (37), there is an item of $\left(d^{2} \varphi_{n} / d x^{2}\right)^{2}$ which can reduce the series' convergence rate. Therefore, it is necessary to consider the influence of high-order modes. The first ten orders data are calculated and listed in Tables 3 and 4.

In this study, we take the random wave in one-year return period $\left(H_{s}=2.04 \mathrm{~m}\right)$ as the excitation. Based on (47), the power spectral density function of the stress resulting from additional displacement was calculated by (37) and plotted in Figure 4; and the power spectral density function of the stress 


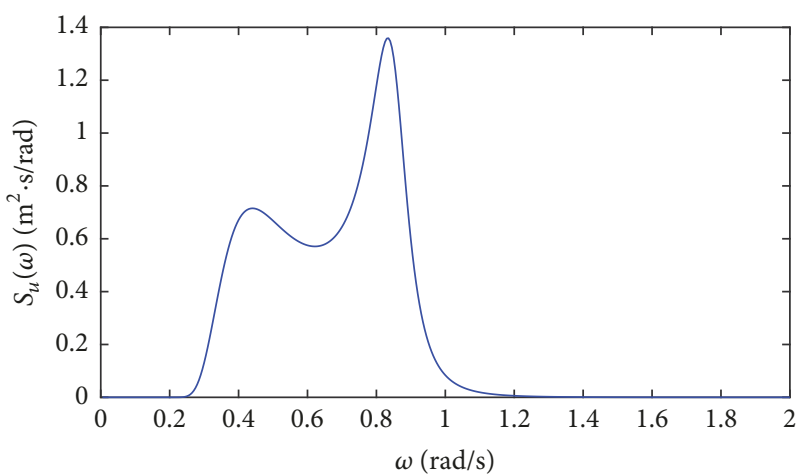

FIGURE 3: The power spectral density function of the platform $\left(H_{s}=\right.$ $10 \mathrm{~m})$.

TABLE 3: Natural frequencies of the riser's lateral vibration.

\begin{tabular}{lcc}
\hline$n$ & $\lambda_{n}$ & $\omega_{n}(\mathrm{rad} / \mathrm{s})$ \\
\hline 1 & 0.0312 & 1.3043 \\
2 & 0.0618 & 5.1004 \\
3 & 0.0910 & 11.0718 \\
4 & 0.1200 & 19.2676 \\
5 & 0.1490 & 29.6696 \\
6 & 0.1776 & 42.1613 \\
7 & 0.2062 & 56.8705 \\
8 & 0.2349 & 73.7973 \\
9 & 0.2636 & 92.8715 \\
10 & 0.2922 & 114.1480 \\
\hline
\end{tabular}

TABLE 4: Weight coefficients of the riser's equivalent load.

\begin{tabular}{lcc}
\hline$n$ & $\delta_{n}$ & $\varepsilon_{n}$ \\
\hline 1 & 0.3478 & $5.99 \times 10^{-5}$ \\
2 & -0.2250 & $1.80 \times 10^{-3}$ \\
3 & 0.1558 & $4.16 \times 10^{-5}$ \\
4 & -0.1175 & $3.32 \times 10^{-5}$ \\
5 & 0.0934 & $2.78 \times 10^{-5}$ \\
6 & -0.0783 & $2.38 \times 10^{-5}$ \\
7 & 0.0673 & $2.07 \times 10^{-5}$ \\
8 & -0.0585 & $1.83 \times 10^{-5}$ \\
9 & 0.0518 & $1.65 \times 10^{-5}$ \\
10 & -0.0534 & $1.72 \times 10^{-5}$ \\
\hline
\end{tabular}

originated from $y_{s}(x, t)$ was computed by (39) and presented in Figure 5. Next, the power spectral density function of the total stress response was obtained by (40) and plotted in Figure 6.

The power spectral density function of the riser has the maximum value when its vibration frequency is equal to the platform's natural frequency. From the comparative analysis between Figures 4 and 6, it can be deduced that the jack-up riser's response mainly comprises the additional displacement response.

Subsequently, the mean square deviation of the riser's random stress response was calculated and depicted in

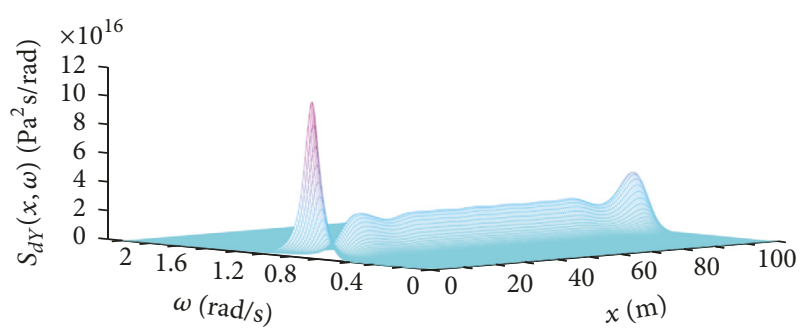

FIGURE 4: PSD of the riser's stress response resulting from additional displacement $\left(H_{s}=2.04 \mathrm{~m}\right)$.

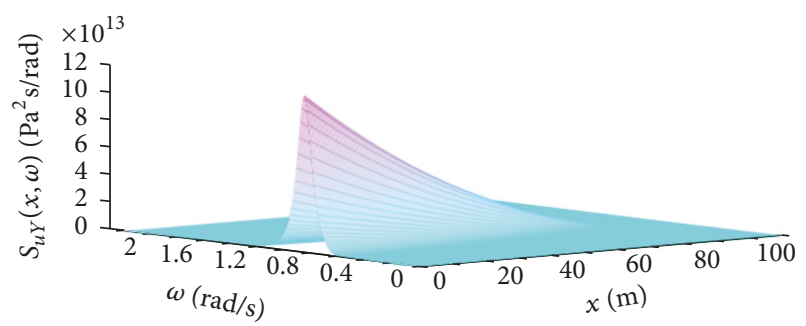

FIGURE 5: PSD of the riser's stress response resulting from quasistatic displacement $\left(H_{s}=2.04 \mathrm{~m}\right)$.

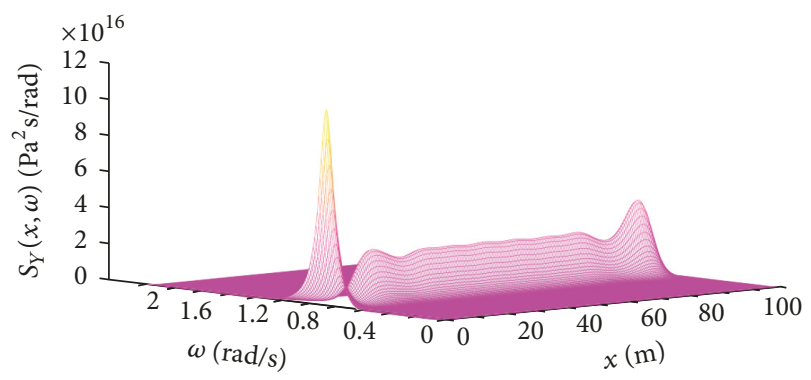

Figure 6: PSD of the riser's stress response $\left(H_{s}=2.04 \mathrm{~m}\right)$.

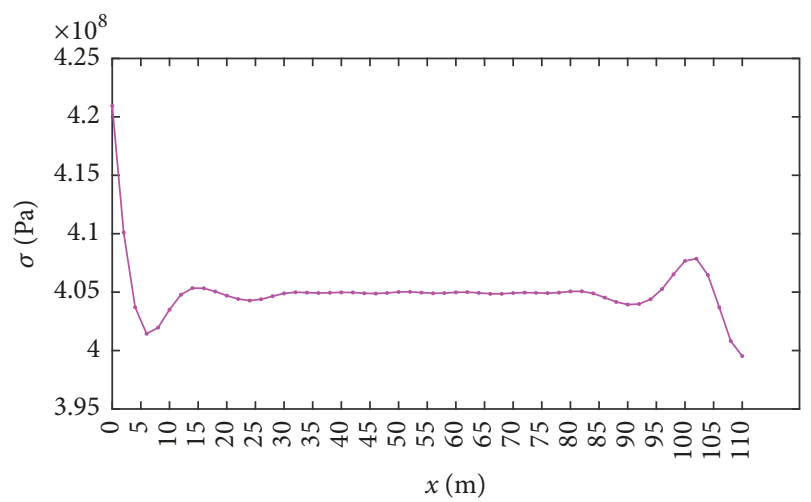

Figure 7: The mean square deviation of the riser's random stress response $\left(H_{s}=2.04 \mathrm{~m}\right)$.

Figure 7. The data shows that the riser has the maximum stress value at its clamped end. In order to determine the type of the riser's random response, the spectral width factor of the riser's stress response was computed by (48). The result shows that the spectral width factor at $x=0 \mathrm{~m}$ is approximately 
TABLE 5: Fatigue life estimation of the riser.

\begin{tabular}{lcc}
\hline \multirow{2}{*}{ Significant wave height $(\mathrm{m})$} & \multicolumn{2}{c}{ Fatigue life (d) } \\
& Bendat method & Steinberg method \\
\hline$H_{s}=0.5$ & $5.08 \times 10^{6}$ & $4.78 \times 10^{6}$ \\
$H_{s}=0.6$ & $1.88 \times 10^{5}$ & $1.77 \times 10^{5}$ \\
$H_{s}=0.7$ & $1.83 \times 10^{4}$ & $1.72 \times 10^{4}$ \\
$H_{s}=0.8$ & $2.65 \times 10^{3}$ & $2.50 \times 10^{3}$ \\
$H_{s}=0.9$ & 495.88 & 466.60 \\
$H_{s}=1.0$ & 114.95 & 108.17 \\
$H_{s}=1.1$ & 32.68 & 30.75 \\
$H_{s}=1.2$ & 11.20 & 10.54 \\
\hline
\end{tabular}

equal to 0.16 (less than 0.3 ); therefore, the riser's response is a narrow band random vibration.

$$
\xi=\sqrt{1-\left(\frac{E[0]}{E[P]}\right)^{2}},
$$

where $\xi$ is the spectral width factor; $E[P]$ is the peak frequency.

$$
E[P]=\frac{1}{2 \pi} \sqrt{\frac{\int_{-\infty}^{\infty} \omega^{4} S_{Y}(x, \omega) d \omega}{\int_{-\infty}^{\infty} \omega^{2} S_{Y}(x, \omega) d \omega}}
$$

Figure 7 shows that the riser's clamped end $(x=0 \mathrm{~m})$ is the most dangerous part. Therefore, the fatigue life of the whole riser system is decided by the fatigue life of the riser's clamped end. In this study, we chose grade B in Table 1 to estimate the riser's fatigue life. The fatigue life of the riser was computed by (42) and (44), respectively, and the data are listed in Table 5. The data in Table 5 shows that the Steinberg method generates the shorter fatigue life; for the sake of safety, the Steinberg method is recommended to estimate the structures' fatigue life. It also can be deduced that the riser in this study cannot be used above level 3 sea condition.

\section{Conclusions}

Based on the Mindlin-Goodman method and the formula proposed by Steinberg, an analytical procedure for the jackup riser's fatigue life estimation is proposed. During the research, we also derived an approximate formula to solve the wave force spectrum.

It is found that the wave loading vibration of jackup riser is a narrow band random vibration. The infinite water depth dispersion relation between wavenumber $k$ and circular frequency $\omega$ is accurate enough to compute the wave force spectrum. The additional displacement response is the main component of the riser's response; and the fatigue life generated by the Steinberg method is less than that of the Bendat method.

The method proposed in this paper can be used to determine whether a special sea area and its sea state are available for a jack-up riser with surface BOP. Besides, the method can be extended to estimate the fatigue life of the Bernoulli-Euler beam with other boundary conditions under random support excitation.

\section{Conflicts of Interest}

The authors declare that they have no conflicts of interest regarding the publication of this paper.

\section{Acknowledgments}

The authors would like to acknowledge the Ministry of Industry and Information Technology of China for supporting this study through the project "Jack-Up Platform Brand Project (II)" (Grant no. 10200001-15-ZC0607-0018).

\section{References}

[1] A. D. Trim, H. Braaten, H. Lie, and M. A. Tognarelli, "Experimental investigation of vortex-induced vibration of long marine risers," Journal of Fluids and Structures, vol. 21, no. 3, pp. 335361, 2005.

[2] H. Xue, W. Tang, and X. Qu, "Prediction and analysis of fatigue damage due to cross-flow and in-line VIV for marine risers in non-uniform current," Ocean Engineering, vol. 83, no. 2, pp. 5262, 2014.

[3] J. Zhang and Y. Tang, "Fatigue analysis of deep-water risers under vortex-induced vibration considering parametric excitations," Journal of Coastal Research, vol. 73, pp. 652-659, 2015.

[4] Y. M. Low and N. Srinil, "VIV fatigue reliability analysis of marine risers with uncertainties in the wake oscillator model," Engineering Structures, vol. 106, pp. 96-108, 2016.

[5] J. Xu, D. Wang, H. Huang, M. Duan, J. Gu, and C. An, "A vortexinduced vibration model for the fatigue analysis of a marine drilling riser," Ships and Offshore Structures, vol. 12, supplement 1, pp. S280-S287, 2017.

[6] Y. M. Low, "Extending a time/frequency domain hybrid method for riser fatigue analysis," Applied Ocean Research, vol. 33, no. 2, pp. 79-87, 2011.

[7] F. Z. Li and Y. M. Low, "Influence of low-frequency vessel motions on the fatigue response of steel catenary risers at the touchdown point," Ships and Offshore Structures, vol. 9, no. 2, pp. 134-148, 2014.

[8] H. Elosta, S. Huang, and A. Incecik, "Wave loading fatigue reliability and uncertainty analyses for geotechnical pipeline models," Ships and Offshore Structures, vol. 9, no. 4, pp. 450-463, 2014.

[9] Y. M. Low, "A variance reduction technique for long-term fatigue analysis of offshore structures using Monte Carlo simulation," Engineering Structures, vol. 128, pp. 283-295, 2016.

[10] G. A. Nothmann, "Vibration of a cantilever beam with prescribed end motion," Transactions of ASME: Journal of Applied Mechanics, vol. 15, pp. 327-334, 1948.

[11] T. C. Yen and S. Kao, "Vibration of a beam-mass system with time-dependent boundary conditions," Transactions of ASME: Journal of Applied Mechanics, vol. 26, pp. 353-356, 1959.

[12] S. Y. Lee and S. M. Lin, "Dynamic analysis of nonuniform beams with time-dependent elastic boundary conditions," Transactions of ASME: Journal of Applied Mechanics, vol. 63, no. 2, pp. 474-478, 1996.

[13] S. Y. Lee and S. M. Lin, "Non-uniform timoshenko beams with time-dependent elastic boundary conditions," Journal of Sound and Vibration, vol. 217, no. 2, pp. 223-238, 1998.

[14] S. M. Lin and S. Y. Lee, "The forced vibration and boundary control of pretwisted timoshenko beams with general time 
dependent elastic boundary conditions," Journal of Sound and Vibration, vol. 254, no. 1, pp. 69-90, 2002.

[15] M. Li, "Analytical study on the dynamic response of a beam with axial force subjected to generalized support excitations," Journal of Sound and Vibration, vol. 338, pp. 199-216, 2015.

[16] Y.-W. Kim, "Dynamic analysis of Timoshenko beam subjected to support motions," Journal of Mechanical Science and Technology, vol. 30, no. 9, pp. 4167-4176, 2016.

[17] R. D. Mindlin and L. E. Goodman, "Beam vibrations with timedependent boundary conditions," Journal of Applied Mechanics, vol. 17, no. 4, pp. 377-380, 1950.

[18] S. Caddemi and I. Caliò, "The influence of the axial force on the vibration of the Euler-Bernoulli beam with an arbitrary number of cracks," Archive of Applied Mechanics, vol. 82, no. 6, pp. 827839, 2012.

[19] Y. Yesilce, "Free and forced vibrations of an axially-loaded Timoshenko multi-span beam carrying a number of various concentrated elements," Shock and Vibration, vol. 19, no. 4, pp. 735-752, 2012.

[20] J. Murin, M. Aminbaghai, V. Kutis, and J. Hrabovsky, "Modal analysis of the FGM beams with effect of axial force under longitudinal variable elastic Winkler foundation," Engineering Structures, vol. 49, pp. 234-247, 2013.

[21] L. E. Borgman, "Spectral analysis of ocean wave forces on piling," Journal of the Waterways, Harbor and Coastal Engineering Division, ASCE, vol. 83, no. 2, pp. 129-156, 1967.

[22] R. W. Clough and J. Penzien, Eds., Dynamics of Structures, Computers \& Structures, University Ave, 3rd edition, 1995.

[23] J. S. Bendat, "Probability functions for random responses," NASA report on Contact NASA-5-4590, 1964.

[24] D. S. Steinberg, Vibration Analysis for Electronic Equipment, John Wiley \& Sons, New York, NY, USA, 2nd edition, 1988.

[25] Y. G. Tang, G. G. Shen, and L. Q. Liu, Hydrodynamics of Marine Structures, Tianjin University Press, Tianjin, China, 2008. 


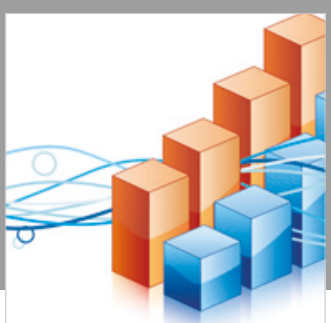

Advances in

Operations Research

\section{-n-m}
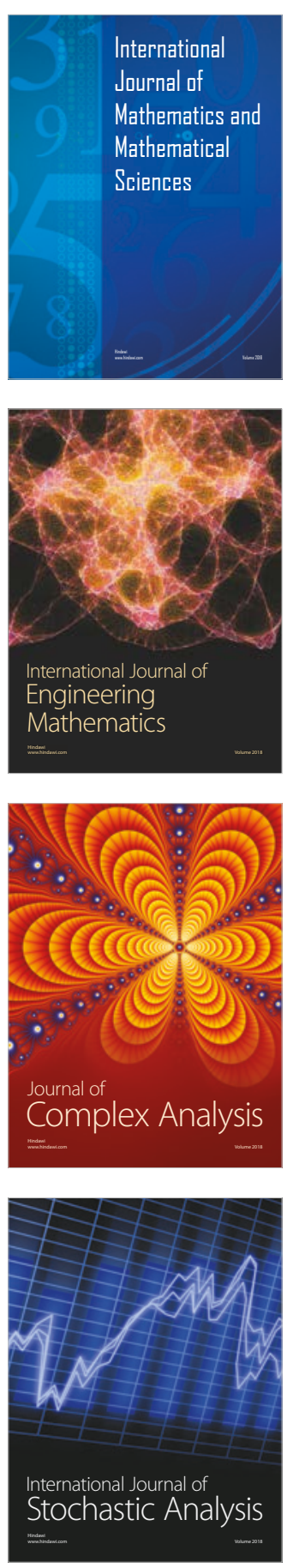
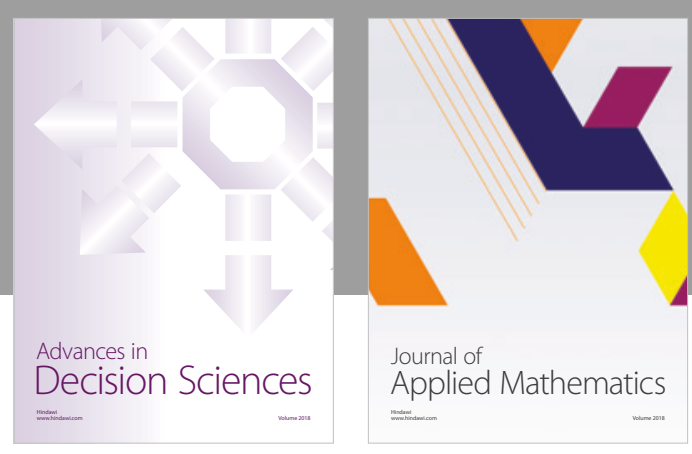

Journal of

Applied Mathematics
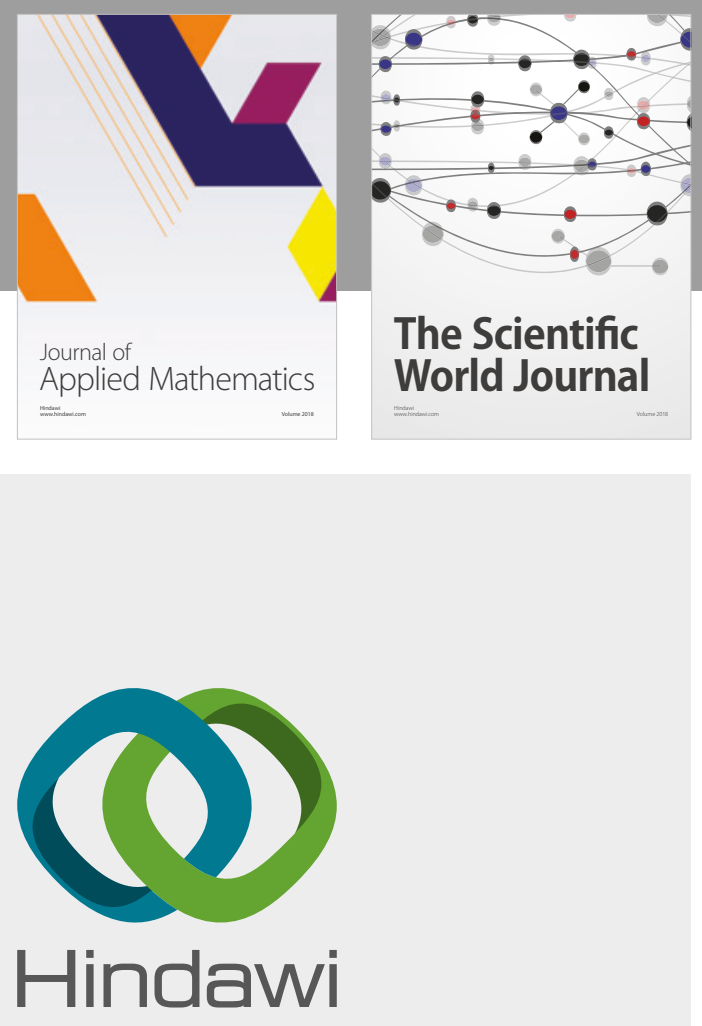

Submit your manuscripts at

www.hindawi.com

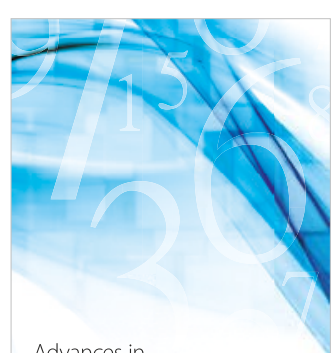

Advances in
Numerical Analysis
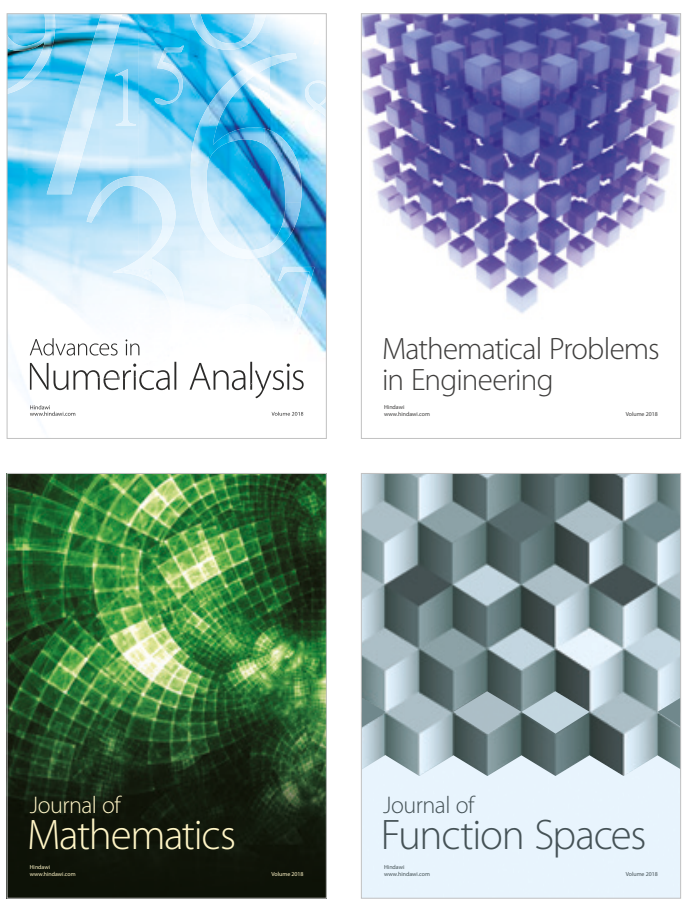

Mathematical Problems in Engineering

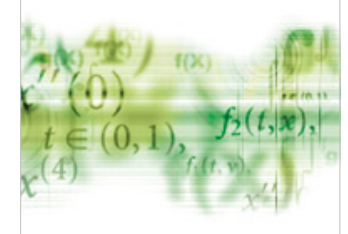

International Journal of

Differential Equations

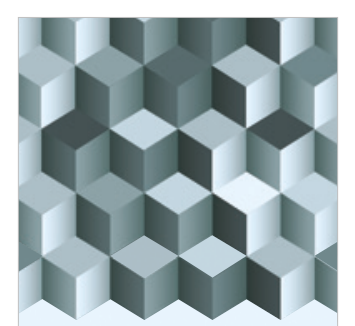

Journal of

Function Spaces

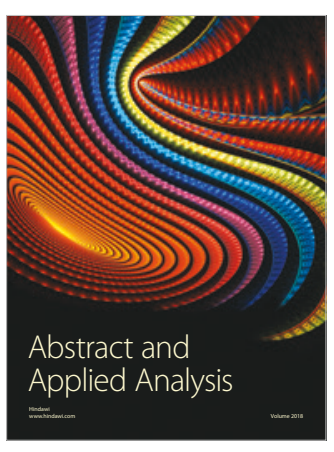

The Scientific

World Journal

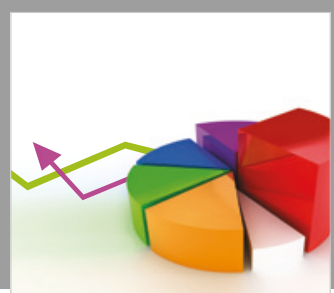

Journal of

Probability and Statistics
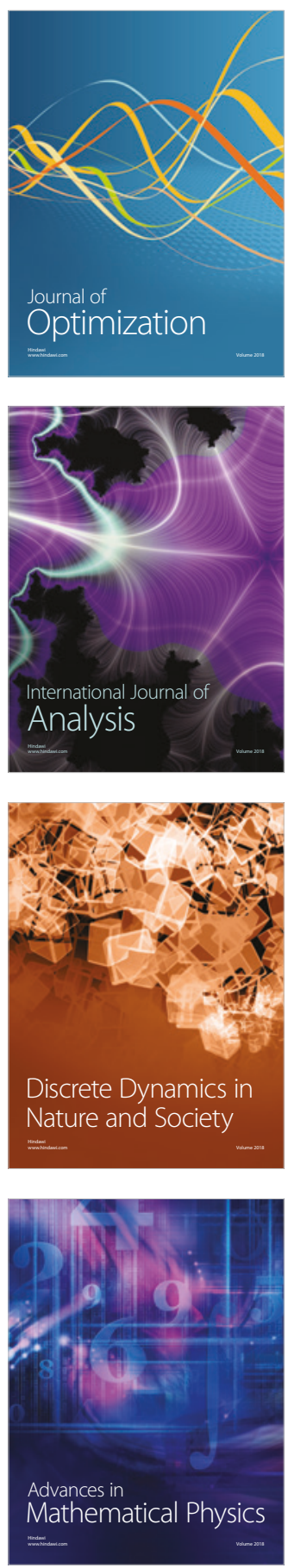